\title{
ECCENTRIC PHASE
}

Factores intra-sujetos

Medida:MEASURE_1

\begin{tabular}{|c|l|}
\hline Tiempo & \multicolumn{1}{c|}{ Variable dependiente } \\
\hline 1 & G_CMJ_Med_Pre_Tiempo_EXCENTRICO_repetición \\
2 & G_CMJ_10_Tiempo_EXCENTRICO_repetición \\
3 & G_CMJ_Med_Post_Tiempo_EXCENTRICO_repetición \\
\hline
\end{tabular}

\begin{tabular}{|l|r|r|r|}
\hline \multicolumn{2}{|c|}{ Estadísticos descriptivos } \\
\hline G_CMJ_Med_Pre_Tiempo_EXCENTRICO_repetición & Media & Desviación típica & N \\
\hline G_CMJ_10_Tiempo_EXCENTRICO_repetición &, 243882 &, 0799648 & 34 \\
G_CMJ_Med_Post_Tiempo_EXCENTRICO_repetición &, 510431 &, 0891227 & 34 \\
\end{tabular}

\begin{tabular}{|c|c|c|c|c|c|c|c|c|c|}
\hline \multicolumn{10}{|c|}{ Contrastes multivariados $^{c}$} \\
\hline Efecto & & Valor & $F$ & $\begin{array}{l}\text { Gl de la } \\
\text { hipótesis }\end{array}$ & Gl del error & Sig. & $\begin{array}{c}\text { Eta al cuadrado } \\
\text { parcial }\end{array}$ & $\begin{array}{c}\text { Parámetro de } \\
\text { no centralidad } \\
\text { Parámetro }\end{array}$ & $\begin{array}{c}\text { Potencia } \\
\text { observada }^{b}\end{array}$ \\
\hline \multirow[t]{4}{*}{ Tiempo } & Traza de Pillai & ,866 & $103,273^{a}$ & 2,000 & 32,000 & ,000 & ,866 & 206,545 & 1,000 \\
\hline & Lambda de Wilks & ,134 & $103,273^{a}$ & 2,000 & 32,000 &, 000 & ,866 & 206,545 & 1,000 \\
\hline & Traza de Hotelling & 6,455 & $103,273^{\mathrm{a}}$ & 2,000 & 32,000 & ,000 & ,866 & 206,545 & 1,000 \\
\hline & Raíz mayor de Roy & 6,455 & $103,273^{a}$ & 2,000 & 32,000 &, 000 & ,866 & 206,545 & 1,000 \\
\hline
\end{tabular}

a. Estadístico exacto

b. Calculado con alfa $=, 05$

c. Diseño: Intersección

Diseño intra-sujetos: Tiempo 
Prueba de esfericidad de Mauchly

Medida:MEASURE_1
\begin{tabular}{|r|r|r|r|r|r|r|r|}
\hline Efecto intra-sujetos & & Chi-cuadrado & & & \multicolumn{3}{|c|}{ Epsilon $^{\text {a }}$} \\
\cline { 5 - 9 } & W de Mauchly & gl & Sig. & $\begin{array}{c}\text { Greenhouse- } \\
\text { Geisser }\end{array}$ & Huynh-Feldt & Límite-inferior \\
\hline Tiempo &, 790 & 7,553 & 2 &, 023 &, 826 &, 864 &, 500 \\
\hline
\end{tabular}

Contrasta la hipótesis nula de que la matriz de covarianza error de las variables dependientes transformadas es proporcional a una matriz identidad.

a. Puede usarse para corregir los grados de libertad en las pruebas de significación promediadas. Las pruebas corregidas se muestran

en la tabla Pruebas de los efectos inter-sujetos.

b. Diseño: Intersección

Diseño intra-sujetos: Tiempo

Pruebas de efectos intra-sujetos.

\begin{tabular}{|c|c|c|c|c|c|c|c|c|c|}
\hline Origen & & $\begin{array}{c}\text { Suma de } \\
\text { cuadrados tipo } \\
\text { III }\end{array}$ & gl & $\begin{array}{c}\text { Media } \\
\text { cuadrática }\end{array}$ & $\mathrm{F}$ & Sig. & $\begin{array}{c}\text { Eta al cuadrado } \\
\text { parcial }\end{array}$ & $\begin{array}{c}\text { Parámetro de } \\
\text { no centralidad } \\
\text { Parámetro }\end{array}$ & $\begin{array}{c}\text { Potencia } \\
\text { observada }^{a}\end{array}$ \\
\hline \multirow[t]{4}{*}{ Tiempo } & Esfericidad asumida & 1,492 & 2 & ,746 & 113,924 &, 000 & ,775 & 227,848 & 1,000 \\
\hline & Greenhouse-Geisser & 1,492 & 1,653 & ,903 & 113,924 &, 000 & ,775 & 188,267 & 1,000 \\
\hline & Huynh-Feldt & 1,492 & 1,729 & ,863 & 113,924 &, 000 & ,775 & 196,930 & 1,000 \\
\hline & Límite-inferior & 1,492 & 1,000 & 1,492 & 113,924 &, 000 & ,775 & 113,924 & 1,000 \\
\hline \multirow[t]{4}{*}{ Error(Tiempo) } & Esfericidad asumida & ,432 & 66 &, 007 & & & & & \\
\hline & Greenhouse-Geisser & ,432 & 54,535 &, 008 & & & & & \\
\hline & Huynh-Feldt & ,432 & 57,044 & ,008 & & & & & \\
\hline & Límite-inferior & ,432 & 33,000 & ,013 & & & & & \\
\hline
\end{tabular}

a. Calculado con alfa $=, 05$ 
Comparaciones por pares

\begin{tabular}{|c|c|c|c|c|c|c|}
\hline \multirow[t]{2}{*}{ (I)Tiempo } & \multirow[t]{2}{*}{ (J)Tiempo } & \multirow{2}{*}{$\begin{array}{l}\text { Diferencia de } \\
\text { medias (I-J) }\end{array}$} & \multirow[b]{2}{*}{ Error típ. } & \multirow[b]{2}{*}{ Sig. ${ }^{a}$} & \multicolumn{2}{|c|}{$\begin{array}{c}\text { Intervalo de confianza al } 95 \% \\
\text { para la diferencia }\end{array}$} \\
\hline & & & & & Límite inferior & Límite superior \\
\hline \multirow[t]{2}{*}{1} & 2 & ,245* & ,017 & ,000 & ,202 & ,288 \\
\hline & 3 &,- 021 &, 017 & ,699 &,- 065 & ,023 \\
\hline \multirow[t]{2}{*}{2} & 1 &,$- 245^{*}$ & ,017 & ,000 & -288 & -202 \\
\hline & 3 &,$- 267^{*}$ & ,024 & ,000 &,- 326 & -207 \\
\hline \multirow[t]{2}{*}{3} & 1 & ,021 & ,017 & ,699 &,- 023 & ,065 \\
\hline & -2 & ,267* & ,024 &, 000 & ,207 & ,326 \\
\hline
\end{tabular}

Basadas en las medias marginales estimadas.

*. La diferencia de medias es significativa al nivel ,05.

a. Ajuste para comparaciones múltiples: Bonferroni. 


\section{ISOMETRIC PHASE}

\section{Factores intra-sujetos}

Medida:MEASURE 1

\begin{tabular}{|c|l|}
\hline Tiempo & \multicolumn{1}{|c|}{ Variable dependiente } \\
\hline 1 & G_CMJ_Med_Pre_Tiempo_ISOMÉTRICO_repetición \\
2 & G_CMJ_10_Tiempo_ISOMÉTRICO_repetición \\
3 & G_CMJ_Med_Post_Tiempo_ISOMÉTRICO_repetición \\
\hline
\end{tabular}

\section{Estadísticos descriptivos}

\begin{tabular}{|l|r|r|r|}
\hline & Media & Desviación típica & N \\
\hline G_CMJ_Med_Pre_Tiempo_ISOMÉTRICO_repetición &, 009804 &, 0044329 & 34 \\
G_CMJ_10_Tiempo_ISOMÉTRICO_repetición &, 010882 &, 0054203 & 34 \\
G_CMJ_Med_Post_Tiempo_ISOMÉTRICO_repetición &, 010941 &, 0039496 & 34 \\
\hline
\end{tabular}

Contrastes multivariados ${ }^{c}$

\begin{tabular}{|c|c|c|c|c|c|c|c|c|c|}
\hline Efecto & & Valor & $\mathrm{F}$ & $\begin{array}{l}\text { Gl de la } \\
\text { hipótesis }\end{array}$ & Gl del error & Sig. & $\begin{array}{c}\text { Eta al cuadrado } \\
\text { parcial }\end{array}$ & $\begin{array}{l}\text { Parámetro de } \\
\text { no centralidad } \\
\text { Parámetro }\end{array}$ & $\begin{array}{c}\text { Potencia } \\
\text { observada }^{\text {b }}\end{array}$ \\
\hline \multirow[t]{4}{*}{ Tiempo } & Traza de Pillai &, 058 &, $986^{a}$ & 2,000 & 32,000 & ,384 &, 058 & 1,972 & ,206 \\
\hline & Lambda de Wilks & ,942 &, $986^{a}$ & 2,000 & 32,000 & ,384 &, 058 & 1,972 & ,206 \\
\hline & Traza de Hotelling & 062 &, $986^{a}$ & 2,000 & 32,000 & ,384 &, 058 & 1,972 & 206 \\
\hline & Raíz mayor de Roy &, 062 &, $986^{\mathrm{a}}$ & 2,000 & 32,000 & ,384 & ,058 & 1,972 & 206 \\
\hline
\end{tabular}

a. Estadístico exacto

b. Calculado con alfa $=, 05$

c. Diseño: Intersección

Diseño intra-sujetos: Tiempo 
Prueba de esfericidad de Mauchly

Medida:MEASURE_1
\begin{tabular}{|r|r|r|r|r|r|r|r|}
\hline Efecto intra-sujetos & & & & & \multicolumn{3}{|c|}{ Epsilon $^{\text {a }}$} \\
\cline { 6 - 9 } & W de Mauchly & $\begin{array}{c}\text { Chi-cuadrado } \\
\text { aprox. }\end{array}$ & gl & Sig. & $\begin{array}{c}\text { Greenhouse- } \\
\text { Geisser }\end{array}$ & Huynh-Feldt & Límite-inferior \\
\hline Tiempo &, 973 &, 875 & 2 &, 646 &, 974 & 1,000 &, 500 \\
\hline
\end{tabular}

Contrasta la hipótesis nula de que la matriz de covarianza error de las variables dependientes transformadas es proporcional a una matriz identidad.

a. Puede usarse para corregir los grados de libertad en las pruebas de significación promediadas. Las pruebas corregidas se muestran

en la tabla Pruebas de los efectos inter-sujetos.

b. Diseño: Intersección

Diseño intra-sujetos: Tiempo

Pruebas de efectos intra-sujetos.

Medida:MEASURE 1

\begin{tabular}{|c|c|c|c|c|c|c|c|c|c|}
\hline Origen & & $\begin{array}{c}\text { Suma de } \\
\text { cuadrados tipo } \\
\text { III }\end{array}$ & gl & $\begin{array}{c}\text { Media } \\
\text { cuadrática }\end{array}$ & $\mathrm{F}$ & Sig. & $\begin{array}{c}\text { Eta al cuadrado } \\
\text { parcial }\end{array}$ & $\begin{array}{c}\text { Parámetro de } \\
\text { no centralidad } \\
\text { Parámetro }\end{array}$ & $\begin{array}{c}\text { Potencia } \\
\text { observada }^{\mathrm{a}}\end{array}$ \\
\hline \multirow[t]{4}{*}{ Tiempo } & Esfericidad asumida & $2,788 \mathrm{E}-5$ & 2 & $1,394 \mathrm{E}-5$ & 1,019 & ,367 & ,030 & 2,038 & ,221 \\
\hline & Greenhouse-Geisser & $2,788 \mathrm{E}-5$ & 1,947 & $1,431 \mathrm{E}-5$ & 1,019 & ,365 &, 030 & 1,985 & ,218 \\
\hline & Huynh-Feldt & $2,788 \mathrm{E}-5$ & 2,000 & $1,394 \mathrm{E}-5$ & 1,019 & ,367 & ,030 & 2,038 & ,221 \\
\hline & Límite-inferior & $2,788 \mathrm{E}-5$ & 1,000 & $2,788 \mathrm{E}-5$ & 1,019 & ,320 &, 030 & 1,019 &, 165 \\
\hline \multirow[t]{4}{*}{ Error(Tiempo) } & Esfericidad asumida &, 001 & 66 & $1,368 \mathrm{E}-5$ & & & & & \\
\hline & Greenhouse-Geisser & ,001 & 64,267 & $1,405 \mathrm{E}-5$ & & & & & \\
\hline & Huynh-Feldt & ,001 & 66,000 & $1,368 \mathrm{E}-5$ & & & & & \\
\hline & Límite-inferior & 001 & 33,000 & $2,736 \mathrm{E}-5$ & & & & & \\
\hline
\end{tabular}

a. Calculado con alfa $=, 05$ 


\section{Comparaciones por pares}

Medida:MEASURE_1

\begin{tabular}{|c|c|c|c|c|c|c|}
\hline \multirow[t]{2}{*}{ (I)Tiempo } & \multirow[t]{2}{*}{ (J)Tiempo } & \multirow{2}{*}{$\begin{array}{l}\text { Diferencia de } \\
\text { medias (I-J) }\end{array}$} & \multirow[b]{2}{*}{ Error típ. } & \multirow[b]{2}{*}{ Sig. ${ }^{a}$} & \multicolumn{2}{|c|}{$\begin{array}{c}\text { Intervalo de confianza al } 95 \% \\
\text { para la diferencia }^{a}\end{array}$} \\
\hline & & & & & Límite inferior & Límite superior \\
\hline \multirow[t]{2}{*}{1} & 2 &,- 001 & ,001 & ,816 &,- 004 & ,001 \\
\hline & 3 &,- 001 &, 001 &, 561 &,- 003 & ,001 \\
\hline \multirow[t]{2}{*}{2} & 1 & ,001 & ,001 & ,816 &,- 001 & ,004 \\
\hline & -3 & $-5,882 E-5$ &, 001 & 1,000 &,- 002 &, 002 \\
\hline \multirow[t]{2}{*}{3} & 1 & ,001 & ,001 &, 561 &,- 001 & ,003 \\
\hline & -2 & $5,882 \mathrm{E}-5$ &, 001 & 1,000 &,- 002 & ,002 \\
\hline
\end{tabular}

Basadas en las medias marginales estimadas.

a. Ajuste para comparaciones múltiples: Bonferroni. 


\section{CONCENTRIC PHASE}

\section{Factores intra-sujetos}

Medida:MEASURE 1

\begin{tabular}{|l|l|}
\hline Tiempo & \multicolumn{1}{|c|}{ Variable dependiente } \\
\hline 1 & G_CMJ_Med_Pre_Tiempo_CONCÉNTRICO_repetición \\
2 & G_CMJ_10_Tiempo_CONCÉNTRICO_repetición \\
3 & G_CMJ_Med_Post_Tiempo_CONCÉNTRICO_repetición \\
\hline
\end{tabular}

Estadísticos descriptivos

\begin{tabular}{|l|r|r|r|}
\hline & Media & Desviación típica & N \\
\hline G_CMJ_Med_Pre_Tiempo_CONCÉNTRICO_repetición &, 222882 &, 0425357 & 34 \\
G_CMJ_10_Tiempo_CONCÉNTRICO_repetición &, 223882 &, 0597671 & 34 \\
G_CMJ_Med_Post_Tiempo_CONCÉNTRICO_repetición &, 196627 &, 0568130 & 34 \\
\hline
\end{tabular}

\begin{tabular}{|c|c|c|c|c|c|c|c|c|c|}
\hline \multicolumn{10}{|c|}{ Contrastes multivariados $^{c}$} \\
\hline Efecto & & Valor & $F$ & $\begin{array}{l}\text { Gl de la } \\
\text { hipótesis }\end{array}$ & Gl del error & Sig. & $\begin{array}{c}\text { Eta al cuadrado } \\
\text { parcial }\end{array}$ & $\begin{array}{c}\text { Parámetro de } \\
\text { no centralidad } \\
\text { Parámetro }\end{array}$ & $\begin{array}{c}\text { Potencia } \\
\text { observada }^{\text {b }}\end{array}$ \\
\hline \multirow[t]{4}{*}{ Tiempo } & Traza de Pillai & ,425 & $11,836^{\mathrm{a}}$ & 2,000 & 32,000 & ,000 & ,425 & 23,673 & ,990 \\
\hline & Lambda de Wilks &, 575 & $11,836^{a}$ & 2,000 & 32,000 & ,000 & ,425 & 23,673 & ,990 \\
\hline & Traza de Hotelling & ,740 & $11,836^{a}$ & 2,000 & 32,000 & ,000 & ,425 & 23,673 & ,990 \\
\hline & Raíz mayor de Roy & ,740 & $11,836^{a}$ & 2,000 & 32,000 &, 000 & ,425 & 23,673 & ,990 \\
\hline
\end{tabular}

a. Estadístico exacto

b. Calculado con alfa $=, 05$

c. Diseño: Intersección

Diseño intra-sujetos: Tiempo 
Prueba de esfericidad de Mauchly ${ }^{b}$

\begin{tabular}{|c|c|c|c|c|c|c|c|}
\hline \multirow[t]{2}{*}{ Efecto intra-sujetos } & \multirow[b]{2}{*}{ W de Mauchly } & \multirow[b]{2}{*}{$\begin{array}{l}\text { Chi-cuadrado } \\
\text { aprox. }\end{array}$} & \multirow[b]{2}{*}{$\mathrm{gl}$} & \multirow[b]{2}{*}{ Sig. } & \multicolumn{3}{|c|}{ Epsilon $^{a}$} \\
\hline & & & & & $\begin{array}{c}\text { Greenhouse- } \\
\text { Geisser }\end{array}$ & Huynh-Feldt & Límite-inferior \\
\hline Tiempo & ,967 & 1,067 & 2 & ,587 & ,968 & 1,000 &, 500 \\
\hline
\end{tabular}

Contrasta la hipótesis nula de que la matriz de covarianza error de las variables dependientes transformadas es proporcional a una matriz identidad.

a. Puede usarse para corregir los grados de libertad en las pruebas de significación promediadas. Las pruebas corregidas se muestran en la tabla Pruebas de los efectos inter-sujetos.

b. Diseño: Intersección

Diseño intra-sujetos: Tiempo

Pruebas de efectos intra-sujetos.

Medida:MEASURE_1

\begin{tabular}{|c|c|c|c|c|c|c|c|c|c|}
\hline Origen & & $\begin{array}{c}\text { Suma de } \\
\text { cuadrados tipo } \\
\text { III }\end{array}$ & gl & $\begin{array}{c}\text { Media } \\
\text { cuadrática }\end{array}$ & $\mathrm{F}$ & Sig. & $\begin{array}{c}\text { Eta al cuadrado } \\
\text { parcial }\end{array}$ & $\begin{array}{l}\text { Parámetro de } \\
\text { no centralidad } \\
\text { Parámetro }\end{array}$ & $\begin{array}{c}\text { Potencia } \\
\text { observada }^{a}\end{array}$ \\
\hline \multirow[t]{4}{*}{ Tiempo } & Esfericidad asumida & ,016 & 2 &, 008 & 10,574 &, 000 & ,243 & 21,148 & ,986 \\
\hline & Greenhouse-Geisser & ,016 & 1,937 &, 008 & 10,574 &, 000 & ,243 & 20,477 & ,984 \\
\hline & Huynh-Feldt & ,016 & 2,000 &, 008 & 10,574 &, 000 & ,243 & 21,148 & ,986 \\
\hline & Límite-inferior & ,016 & 1,000 &, 016 & 10,574 &, 003 & ,243 & 10,574 & ,884 \\
\hline \multirow[t]{4}{*}{ Error(Tiempo) } & Esfericidad asumida & ,051 & 66 &, 001 & & & & & \\
\hline & Greenhouse-Geisser & 051 & 63,905 &, 001 & & & & & \\
\hline & Huynh-Feldt & ,051 & 66,000 &, 001 & & & & & \\
\hline & Límite-inferior & ,051 & 33,000 & ,002 & & & & & \\
\hline
\end{tabular}

a. Calculado con alfa $=, 05$ 


\section{Comparaciones por pares}

\begin{tabular}{|c|c|c|c|c|c|c|}
\hline \multirow[t]{2}{*}{ (I)Tiempo } & \multirow[t]{2}{*}{ (J)Tiempo } & \multirow{2}{*}{$\begin{array}{c}\text { Diferencia de } \\
\text { medias (I-J) }\end{array}$} & \multirow[b]{2}{*}{ Error típ. } & \multirow[b]{2}{*}{ Sig. $^{a}$} & \multicolumn{2}{|c|}{$\begin{array}{c}\text { Intervalo de confianza al } 95 \% \\
\text { para la diferencia }\end{array}$} \\
\hline & & & & & Límite inferior & Límite superior \\
\hline \multirow[t]{2}{*}{1} & 2 &,- 001 & ,007 & 1,000 &,- 019 & ,017 \\
\hline & 3 &, $026^{*}$ &, 006 &, 000 &, 011 &, 042 \\
\hline \multirow[t]{2}{*}{2} & 1 & , 001 & , 007 & 1,000 &,- 017 & ,019 \\
\hline & 3 &, $027^{*}$ & ,007 & ,001 & 010 &, 045 \\
\hline \multirow[t]{2}{*}{3} & 1 &,$- 026^{*}$ & ,006 & , 000 &,- 042 &,- 011 \\
\hline & -2 &,$- 027^{*}$ & ,007 & ,001 &,- 045 &,- 010 \\
\hline
\end{tabular}

Basadas en las medias marginales estimadas.

a. Ajuste para comparaciones múltiples: Bonferroni.

*. La diferencia de medias es significativa al nivel ,05. 


\section{TOTAL DURATION JUMP}

\section{Factores intra-sujetos}

Medida:MEASURE 1

\begin{tabular}{|c|l|}
\hline Tiempo & \multicolumn{1}{|c|}{ Variable dependiente } \\
\hline 1 & G_CMJ_Med_Pre_Tiempo_TOTAL_repetición \\
2 & G_CMJ_10_Tiempo_TOTAL_repetición \\
3 & G_CMJ_Med_Post_Tiempo_TOTAL_repetición \\
\hline
\end{tabular}

Estadísticos descriptivos

\begin{tabular}{|l|r|r|r|}
\hline & \multicolumn{1}{|c|}{ Media } & Desviación típica & \multicolumn{1}{|c|}{$\mathrm{N}$} \\
\hline G_CMJ_Med_Pre_Tiempo_TOTAL_repetición &, 721863 &, 1034697 & 34 \\
G_CMJ_10_Tiempo_TOTAL_repetición &, 478647 &, 1201898 & 34 \\
G_CMJ_Med_Post_Tiempo_TOTAL_repetición &, 718000 &, 1266440 & 34 \\
\hline
\end{tabular}

\begin{tabular}{|c|c|c|c|c|c|c|c|c|c|}
\hline \multicolumn{10}{|c|}{ Contrastes multivariados $^{\mathrm{C}}$} \\
\hline \multicolumn{2}{|l|}{ Efecto } & \multirow{2}{*}{ Valor } & \multirow[b]{2}{*}{$\mathrm{F}$} & \multirow{2}{*}{$\begin{array}{r}\text { Gl de la } \\
\text { hipótesis }\end{array}$} & \multirow[b]{2}{*}{ Gl del error } & \multirow{2}{*}{ Sig. } & \multirow{2}{*}{$\begin{array}{c}\text { Eta al cuadrado } \\
\text { parcial }\end{array}$} & Parámetro de & \multirow{2}{*}{$\begin{array}{c}\text { Potencia } \\
\text { observada }^{\mathrm{b}}\end{array}$} \\
\hline & & & & & & & & Parámetro & \\
\hline \multirow[t]{4}{*}{ Tiempo } & Traza de Pillai & ,828 & $76,804^{a}$ & 2,000 & 32,000 & , 000 & ,828 & 153,607 & 1,000 \\
\hline & Lambda de Wilks & , 172 & $76,804^{a}$ & 2,000 & 32,000 & , 000 & ,828 & 153,607 & 1,000 \\
\hline & Traza de Hotelling & 4,800 & $76,804^{a}$ & 2,000 & 32,000 & , 000 & 828 & 153,607 & 1,000 \\
\hline & Raíz mayor de Roy & 4,800 & $76,804^{a}$ & 2,000 & 32,000 &, 000 & , 828 & 153,607 & 1,000 \\
\hline
\end{tabular}

a. Estadístico exacto

b. Calculado con alfa $=, 05$

c. Diseño: Intersección

Diseño intra-sujetos: Tiempo 


\section{Prueba de esfericidad de Mauchly ${ }^{b}$}

Medida:MEASURE_1

\begin{tabular}{|c|c|c|c|c|c|c|c|}
\hline \multirow[t]{2}{*}{ Efecto intra-sujetos } & \multirow[b]{2}{*}{ W de Mauchly } & \multirow[b]{2}{*}{$\begin{array}{l}\text { Chi-cuadrado } \\
\text { aprox. }\end{array}$} & \multirow[b]{2}{*}{$\mathrm{gl}$} & \multirow[b]{2}{*}{ Sig. } & \multicolumn{3}{|c|}{ Epsilon $^{a}$} \\
\hline & & & & & $\begin{array}{c}\text { Greenhouse- } \\
\text { Geisser }\end{array}$ & Huynh-Feldt & Límite-inferior \\
\hline Tiempo & ,795 & 7,329 & 2 & ,026 & ,830 & ,869 &, 500 \\
\hline
\end{tabular}

Contrasta la hipótesis nula de que la matriz de covarianza error de las variables dependientes transformadas es proporcional a una matriz identidad.

a. Puede usarse para corregir los grados de libertad en las pruebas de significación promediadas. Las pruebas corregidas se muestran en la tabla Pruebas de los efectos inter-sujetos.

b. Diseño: Intersección

Diseño intra-sujetos: Tiempo

Pruebas de efectos intra-sujetos.

Medida:MEASURE_1

\begin{tabular}{|c|c|c|c|c|c|c|c|c|c|}
\hline Origen & & $\begin{array}{c}\text { Suma de } \\
\text { cuadrados tipo } \\
\text { III }\end{array}$ & gl & $\begin{array}{c}\text { Media } \\
\text { cuadrática }\end{array}$ & $\mathrm{F}$ & Sig. & $\begin{array}{c}\text { Eta al cuadrado } \\
\text { parcial }\end{array}$ & $\begin{array}{c}\text { Parámetro de } \\
\text { no centralidad } \\
\text { Parámetro }\end{array}$ & $\begin{array}{c}\text { Potencia } \\
\text { observada }^{a} \\
\end{array}$ \\
\hline \multirow[t]{4}{*}{ Tiempo } & Esfericidad asumida & 1,320 & 2 &, 660 & 86,875 &, 000 & ,725 & 173,749 & 1,000 \\
\hline & Greenhouse-Geisser & 1,320 & 1,660 & ,795 & 86,875 &, 000 & ,725 & 144,228 & 1,000 \\
\hline & Huynh-Feldt & 1,320 & 1,737 & ,760 & 86,875 & ,000 & ,725 & 150,926 & 1,000 \\
\hline & Límite-inferior & 1,320 & 1,000 & 1,320 & 86,875 &, 000 & ,725 & 86,875 & 1,000 \\
\hline \multirow[t]{4}{*}{ Error(Tiempo) } & Esfericidad asumida &, 501 & 66 &, 008 & & & & & \\
\hline & Greenhouse-Geisser &, 501 & 54,786 &, 009 & & & & & \\
\hline & Huynh-Feldt &, 501 & 57,330 &, 009 & & & & & \\
\hline & Límite-inferior &, 501 & 33,000 &, 015 & & & & & \\
\hline
\end{tabular}

a. Calculado con alfa $=, 05$ 


\section{Comparaciones por pares}

Medida:MEASURE_1

\begin{tabular}{|c|c|c|c|c|c|c|}
\hline \multirow[t]{2}{*}{ (I)Tiempo } & \multirow[t]{2}{*}{ (J)Tiempo } & \multirow{2}{*}{$\begin{array}{c}\text { Diferencia de } \\
\text { medias (I-J) } \\
\end{array}$} & \multirow[b]{2}{*}{ Error típ. } & \multirow[b]{2}{*}{ Sig. ${ }^{a}$} & \multicolumn{2}{|c|}{$\begin{array}{c}\text { Intervalo de confianza al } 95 \% \\
\text { para la diferencia }\end{array}$} \\
\hline & & & & & Límite inferior & Límite superior \\
\hline \multirow[t]{2}{*}{1} & 2 & ,243* &, 019 &, 000 & , 194 & ,292 \\
\hline & 3 &, 004 &, 018 & 1,000 &,- 041 &, 049 \\
\hline \multirow[t]{2}{*}{2} & 1 &,$- 243^{*}$ & ,019 & ,000 &,- 292 &,- 194 \\
\hline & 3 &,$- 239^{*}$ &, 025 &, 000 &,- 303 &,- 175 \\
\hline \multirow[t]{2}{*}{3} & 1 &,- 004 & ,018, & 1,000 & -,049 & ,041 \\
\hline & 2 & ,239* & ,025 & ,000 & 175, & ,303, \\
\hline
\end{tabular}

Basadas en las medias marginales estimadas.

*. La diferencia de medias es significativa al nivel ,05.

a. Ajuste para comparaciones múltiples: Bonferroni. 


\section{MAXIMUM FORCE}

\section{Factores intra-sujetos}

Medida:MEASURE_1
\begin{tabular}{|l|l|}
\hline Tiempo & \multicolumn{1}{|c|}{ Variable dependiente } \\
\hline 1 & G_CMJ_Media_Pre_Fmax \\
2 & G_CMJ_10_Fmax \\
3 & G_CMJ_Media_Post_Fmax \\
\hline
\end{tabular}

\begin{tabular}{|c|c|c|c|}
\hline \multicolumn{4}{|c|}{ Estadísticos descriptivos } \\
\hline & Media & $\begin{array}{c}\text { Desviación } \\
\text { típica }\end{array}$ & $\mathrm{N}$ \\
\hline G_CMJ_Media_Pre_Fmax & 2021,6696 & 237,48624 & 34 \\
\hline G_CMJ_10_Fmax & 1973,4015 & 224,22813 & 34 \\
\hline G_CMJ_Media_Post_Fmax & 1934,3988 & 361,17159 & 34 \\
\hline
\end{tabular}

\begin{tabular}{|c|c|c|c|c|c|c|c|c|c|}
\hline \multicolumn{10}{|c|}{ Contrastes multivariados $^{c}$} \\
\hline Efecto & & Valor & $\mathrm{F}$ & $\begin{array}{l}\text { Gl de la } \\
\text { hipótesis }\end{array}$ & Gl del error & Sig. & $\begin{array}{c}\text { Eta al cuadrado } \\
\text { parcial }\end{array}$ & $\begin{array}{l}\text { Parámetro de } \\
\text { no centralidad } \\
\text { Parámetro }\end{array}$ & $\begin{array}{c}\text { Potencia } \\
\text { observada }^{\text {b }}\end{array}$ \\
\hline \multirow[t]{4}{*}{ Tiempo } & Traza de Pillai & , 150 & $2,828^{a}$ & 2,000 & 32,000 & 074 & , 150 & 5,656 &, 516 \\
\hline & Lambda de Wilks & ,850 & $2,828^{a}$ & 2,000 & 32,000 & ,074 & , 150 & 5,656 &, 516 \\
\hline & Traza de Hotelling & , 177 & $2,828^{a}$ & 2,000 & 32,000 & ,074 & , 150 & 5,656 &, 516 \\
\hline & Raíz mayor de Roy & ,177 & $2,828^{\mathrm{a}}$ & 2,000 & 32,000 & ,074 &, 150 & 5,656 &, 516 \\
\hline
\end{tabular}
a. Estadístico exacto
b. Calculado con alfa $=, 05$
c. Diseño: Intersección
Diseño intra-sujetos: Tiempo 


\section{Prueba de esfericidad de Mauchly}

Medida:MEASURE_1

\begin{tabular}{|c|c|c|c|c|c|c|c|}
\hline \multirow[t]{2}{*}{ Efecto intra-sujetos } & \multirow[b]{2}{*}{ W de Mauchly } & \multirow[b]{2}{*}{$\begin{array}{l}\text { Chi-cuadrado } \\
\text { aprox. }\end{array}$} & \multirow[b]{2}{*}{$\mathrm{gl}$} & \multirow[b]{2}{*}{ Sig. } & \multicolumn{3}{|c|}{ Epsilon $^{a}$} \\
\hline & & & & & $\begin{array}{c}\text { Greenhouse- } \\
\text { Geisser }\end{array}$ & Huynh-Feldt & Límite-inferior \\
\hline Tiempo & ,406 & 28,850 & 2 &, 000 & ,627 & 640 &, 500 \\
\hline
\end{tabular}

Contrasta la hipótesis nula de que la matriz de covarianza error de las variables dependientes transformadas es proporcional a una matriz identidad.

a. Puede usarse para corregir los grados de libertad en las pruebas de significación promediadas. Las pruebas corregidas se muestran en la tabla Pruebas de los efectos inter-sujetos.

b. Diseño: Intersección

Diseño intra-sujetos: Tiempo

Pruebas de efectos intra-sujetos.

Medida:MEASURE_1

\begin{tabular}{|c|c|c|c|c|c|c|c|c|c|}
\hline Origen & & $\begin{array}{c}\text { Suma de } \\
\text { cuadrados tipo } \\
\text { III }\end{array}$ & gl & $\begin{array}{c}\text { Media } \\
\text { cuadrática }\end{array}$ & $\mathrm{F}$ & Sig. & $\begin{array}{c}\text { Eta al cuadrado } \\
\text { parcial }\end{array}$ & $\begin{array}{l}\text { Parámetro de } \\
\text { no centralidad } \\
\text { Parámetro }\end{array}$ & $\begin{array}{c}\text { Potencia } \\
\text { observada }^{a} \\
\end{array}$ \\
\hline \multirow[t]{4}{*}{ Tiempo } & Esfericidad asumida & 129961,709 & 2 & 64980,854 & 2,170 & ,122 &, 062 & 4,340 & ,429 \\
\hline & Greenhouse-Geisser & 129961,709 & 1,255 & 103583,546 & 2,170 & ,144 &, 062 & 2,723 & ,334 \\
\hline & Huynh-Feldt & 129961,709 & 1,281 & 101472,001 & 2,170 & ,143 &, 062 & 2,780 & ,338 \\
\hline & Límite-inferior & 129961,709 & 1,000 & 129961,709 & 2,170 &, 150 &, 062 & 2,170 & ,299 \\
\hline \multirow[t]{4}{*}{ Error(Tiempo) } & Esfericidad asumida & 1976184,008 & 66 & 29942,182 & & & & & \\
\hline & Greenhouse-Geisser & 1976184,008 & 41,404 & 47729,711 & & & & & \\
\hline & Huynh-Feldt & 1976184,008 & 42,265 & 46756,743 & & & & & \\
\hline & Límite-inferior & 1976184,008 & 33,000 & 59884,364 & & & & & \\
\hline
\end{tabular}

a. Calculado con alfa $=, 05$ 


\section{Comparaciones por pares}

Medida:MEASURE_1

\begin{tabular}{|c|c|c|c|c|c|c|}
\hline \multirow[t]{2}{*}{ (I)Tiempo } & \multirow[t]{2}{*}{ (J)Tiempo } & \multirow{2}{*}{$\begin{array}{c}\text { Diferencia de } \\
\text { medias (I-J) } \\
\end{array}$} & \multirow[b]{2}{*}{ Error típ. } & \multirow[b]{2}{*}{ Sig. $^{a}$} & \multicolumn{2}{|c|}{$\begin{array}{c}\text { Intervalo de confianza al } 95 \% \\
\text { para la diferencia }{ }^{a}\end{array}$} \\
\hline & & & & & Límite inferior & Límite superior \\
\hline \multirow[t]{2}{*}{1} & 2 & 48,268 & 20,961 & ,083 & $-4,600$ & 101,136 \\
\hline & -3 & 87,271 & 51,862 & ,306 & $-43,537$ & 218,079 \\
\hline \multirow[t]{2}{*}{2} & 1 & $-48,268$ & 20,961 & ,083 & $-101,136$ & 4,600 \\
\hline & -3 & 39,003 & 46,420 & 1,000 & $-78,079$ & 156,085 \\
\hline \multirow[t]{2}{*}{3} & 1 & $-87,271$ & 51,862 & ,306 & $-218,079$ & 43,537 \\
\hline & -2 & $-39,003$ & 46,420 & 1,000 & $-156,085$ & 78,079 \\
\hline
\end{tabular}

Basadas en las medias marginales estimadas.

a. Ajuste para comparaciones múltiples: Bonferroni. 


\section{PEAK RATE OF FORCE DEVELOPMENT}

Factores intra-sujetos

Medida:MEASURE_1
\begin{tabular}{|c|l|}
\hline Tiempo & \multicolumn{1}{|c|}{ Variable dependiente } \\
\hline 1 & G_CMJ_Media_Pre_RFD \\
2 & G_CMJ_10_RFD \\
3 & G_CMJ_Media_Post_RFD \\
\hline
\end{tabular}

\begin{tabular}{|l|c|c|r|}
\hline \multicolumn{1}{|c|}{ Estadísticos descriptivos } \\
& Media & $\begin{array}{c}\text { Desviación } \\
\text { típica }\end{array}$ & N \\
\hline G_CMJ_Media_Pre_RFD & 4235,2400 & 976,99288 & 34 \\
G_CMJ_10_RFD & 4150,3596 & 1113,63632 & 34 \\
G_CMJ_Media_Post_RFD & 3951,3427 & 1266,23782 & 34 \\
\hline
\end{tabular}

\begin{tabular}{|c|c|c|c|c|c|c|c|c|c|}
\hline \multicolumn{10}{|c|}{ Contrastes multivariados $^{c}$} \\
\hline Efecto & & & & & & & & Parámetro de & \\
\hline & & Valor & $\mathrm{F}$ & hipótesis & Gl del error & Sig. & parcial & Parámetro & observada $^{\mathrm{b}}$ \\
\hline \multirow[t]{4}{*}{ Tiempo } & Traza de Pillai &, 080 & $1,395^{\mathrm{a}}$ & 2,000 & 32,000 & ,262 &, 080 & 2,790 & ,277 \\
\hline & Lambda de Wilks & ,920 & $1,395^{\mathrm{a}}$ & 2,000 & 32,000 & ,262 &, 080 & 2,790 & ,277 \\
\hline & Traza de Hotelling &, 087 & $1,395^{\mathrm{a}}$ & 2,000 & 32,000 & ,262 &, 080 & 2,790 & ,277 \\
\hline & Raíz mayor de Roy &, 087 & $1,395^{\mathrm{a}}$ & 2,000 & 32,000 & ,262 &, 080 & 2,790 & 277 \\
\hline
\end{tabular}

a. Estadístico exacto

b. Calculado con alfa $=, 05$

c. Diseño: Intersección

Diseño intra-sujetos: Tiempo 


\section{Prueba de esfericidad de Mauchly}

Medida:MEASURE_1

\begin{tabular}{|c|c|c|c|c|c|c|c|}
\hline \multirow[t]{2}{*}{ Efecto intra-sujetos } & \multirow[b]{2}{*}{ W de Mauchly } & \multirow[b]{2}{*}{$\begin{array}{c}\text { Chi-cuadrado } \\
\text { aprox. }\end{array}$} & \multirow[b]{2}{*}{$\mathrm{gl}$} & \multirow[b]{2}{*}{ Sig. } & \multicolumn{3}{|c|}{ Epsilon $^{\mathrm{a}}$} \\
\hline & & & & & $\begin{array}{c}\text { Greenhouse- } \\
\text { Geisser }\end{array}$ & Huynh-Feldt & Límite-inferior \\
\hline Tiempo & ,919 & 2,706 & 2 & ,259 & ,925 & 978 &, 500 \\
\hline
\end{tabular}

Contrasta la hipótesis nula de que la matriz de covarianza error de las variables dependientes transformadas es proporcional a una matriz identidad.

a. Puede usarse para corregir los grados de libertad en las pruebas de significación promediadas. Las pruebas corregidas se muestran en la tabla Pruebas de los efectos inter-sujetos.

b. Diseño: Intersección

Diseño intra-sujetos: Tiempo

Pruebas de efectos intra-sujetos.

Medida:MEASURE_1

\begin{tabular}{|c|c|c|c|c|c|c|c|c|c|}
\hline Origen & & $\begin{array}{c}\text { Suma de } \\
\text { cuadrados tipo } \\
\text { III }\end{array}$ & $\mathrm{gl}$ & $\begin{array}{c}\text { Media } \\
\text { cuadrática }\end{array}$ & $\mathrm{F}$ & Sig. & $\begin{array}{c}\text { Eta al cuadrado } \\
\text { parcial }\end{array}$ & $\begin{array}{c}\text { Parámetro de } \\
\text { no centralidad } \\
\text { Parámetro }\end{array}$ & $\begin{array}{c}\text { Potencia } \\
\text { observada }^{\mathrm{a}}\end{array}$ \\
\hline \multirow[t]{4}{*}{ Tiempo } & Esfericidad asumida & 1443981,192 & 2 & 721990,596 & 1,846 &, 166 & ,053 & 3,691 & ,372 \\
\hline & Greenhouse-Geisser & 1443981,192 & 1,850 & 780524,304 & 1,846 &, 169 & ,053 & 3,415 & ,356 \\
\hline & Huynh-Feldt & 1443981,192 & 1,955 & 738581,807 & 1,846 &, 167 & ,053 & 3,608 & ,367 \\
\hline & Límite-inferior & 1443981,192 & 1,000 & 1443981,192 & 1,846 & ,183 &, 053 & 1,846 & ,261 \\
\hline \multirow[t]{4}{*}{ Error(Tiempo) } & Esfericidad asumida & $2,582 \mathrm{E} 7$ & 66 & 391173,784 & & & & & \\
\hline & Greenhouse-Geisser & $2,582 \mathrm{E} 7$ & 61,050 & 422887,288 & & & & & \\
\hline & Huynh-Feldt & $2,582 \mathrm{E} 7$ & 64,517 & 400162,886 & & & & & \\
\hline & Límite-inferior & $2,582 \mathrm{E} 7$ & 33,000 & 782347,568 & & & & & \\
\hline
\end{tabular}

a. Calculado con alfa $=, 05$ 


\section{Comparaciones por pares}

Medida:MEASURE_1

\begin{tabular}{|c|c|c|c|c|c|c|}
\hline \multirow[t]{2}{*}{ (I)Tiempo } & \multirow[t]{2}{*}{ (J)Tiempo } & \multirow{2}{*}{$\begin{array}{l}\text { Diferencia de } \\
\text { medias (I-J) }\end{array}$} & \multirow[b]{2}{*}{ Error típ. } & \multirow[b]{2}{*}{ Sig. ${ }^{a}$} & \multicolumn{2}{|c|}{$\begin{array}{c}\text { Intervalo de confianza al } 95 \% \\
\text { para la diferencia }^{a}\end{array}$} \\
\hline & & & & & Límite inferior & Límite superior \\
\hline \multirow[t]{2}{*}{1} & 2 & 84,880 & 133,764 & 1,000 & $-252,502$ & 422,263 \\
\hline & 3 & 283,897 & 170,623 & ,317 & $-146,449$ & 714,244 \\
\hline \multirow[t]{2}{*}{2} & 1 & $-84,880$ & 133,764 & 1,000 & $-422,263$ & 252,502 \\
\hline & -3 & 199,017 & 148,411 &, 567 & $-175,306$ & 573,340 \\
\hline \multirow[t]{2}{*}{3} & 1 & $-283,897$ & 170,623 & ,317 & $-714,244$ & 146,449 \\
\hline & -2 & $-199,017$ & 148,411 & ,567 & $-573,340$ & 175,306 \\
\hline
\end{tabular}

Basadas en las medias marginales estimadas.

a. Ajuste para comparaciones múltiples: Bonferroni. 